\title{
Similarity-Based Models of Word Cooccurrence Probabilities
}

IDO DAGAN

dagan@macs.biu.ac.il

Dept. of Mathematics and Computer Science, Bar Ilan University, Ramat Gan 52900, Israel

LILLIAN LEE

llee@cs.cornell.edu

Department of Computer Science, Cornell University, Ithaca, NY 14853, USA

FERNANDO C. N. PEREIRA

pereira@research.att.com

AT\&T Labs - Research, 180 Park Ave., Florham Park, NJ 07932, USA

Editors: Claire Cardie and Raymond Mooney

\begin{abstract}
In many applications of natural language processing (NLP) it is necessary to determine the likelihood of a given word combination. For example, a speech recognizer may need to determine which of the two word combinations "eat a peach" and "eat a beach" is more likely. Statistical NLP methods determine the likelihood of a word combination from its frequency in a training corpus. However, the nature of language is such that many word combinations are infrequent and do not occur in any given corpus. In this work we propose a method for estimating the probability of such previously unseen word combinations using available information on "most similar" words.

We describe probabilistic word association models based on distributional word similarity, and apply them to two tasks, language modeling and pseudo-word disambiguation. In the language modeling task, a similarity-based model is used to improve probability estimates for unseen bigrams in a back-off language model. The similaritybased method yields a $20 \%$ perplexity improvement in the prediction of unseen bigrams and statistically significant reductions in speech-recognition error.

We also compare four similarity-based estimation methods against back-off and maximum-likelihood estimation methods on a pseudo-word sense disambiguation task in which we controlled for both unigram and bigram frequency to avoid giving too much weight to easy-to-disambiguate high-frequency configurations. The similaritybased methods perform up to $40 \%$ better on this particular task.
\end{abstract}

Keywords: Statistical language modeling; sense disambiguation

\section{Introduction}

Data sparseness is an inherent problem in statistical methods for natural language processing. Such methods use statistics on the relative frequencies of configurations of elements in a training corpus to learn how to evaluate alternative analyses or interpretations of new samples of text or speech. The most likely analysis will be taken to be the one that contains the most frequent configurations. The problem of data sparseness, also known as the zero-frequency problem (Witten \& Bell, 1991), arises when analyses contain configurations that never occurred in the training corpus. Then it is not possible to estimate probabilities from observed frequencies, and some other estimation scheme that can generalize from the training data has to be used.

In language processing applications, the sparse data problem occurs even for very large data sets. For example, Essen \& Steinbiss (1992) report that in a 75\%-25\% split of the million-word LOB corpus, $12 \%$ of the bigrams in the test partition did not occur in the 
training portion. For trigrams, the sparse data problem is even more severe: for instance, researchers at IBM (Brown, DellaPietra, deSouza, Lai, \& Mercer, 1992) examined a training corpus consisting of almost 366 million English words, and discovered that one can expect $14.7 \%$ of the word triples in any new English text to be absent from the training sample. Thus, estimating the probability of unseen configurations is crucial to accurate language modeling, since the aggregate probability of these unseen events can be significant.

We focus here on a particular kind of configuration, word cooccurrence. Examples of such cooccurrences include relationships between head words in syntactic constructions (verb-object or adjective-noun, for instance) and word sequences ( $n$-grams). In commonly used models, the probability estimate for a previously unseen cooccurrence is a function of the probability estimates for the words in the cooccurrence. For example, in word bigram models, the probability $P\left(w_{2} \mid w_{1}\right)$ of a conditioned word $w_{2}$ that has never occurred in training following the conditioning word $w_{1}$ is typically calculated from the probability of $w_{2}$, as estimated by $w_{2}$ 's frequency in the corpus (Jelinek, Mercer, \& Roukos, 1992; Katz, 1987). This method makes an independence assumption on the cooccurrence of $w_{1}$ and $w_{2}$ : the more frequent $w_{2}$ is, the higher the estimate of $P\left(w_{2} \mid w_{1}\right)$ will be, regardless of $w_{1}$.

Class-based and similarity-based models provide an alternative to the independence assumption. In these models, the relationship between given words is modeled by analogy with other words that are in some sense similar to the given ones.

For instance, Brown et al. (1992) suggest a class-based $n$-gram model in which words with similar cooccurrence distributions are clustered into word classes. The cooccurrence probability of a given pair of words is then estimated according to an averaged cooccurrence probability of the two corresponding classes. Pereira, Tishby, \& Lee (1993) propose a "soft" distributional clustering scheme for certain grammatical cooccurrences in which membership of a word in a class is probabilistic. Cooccurrence probabilities of words are then modeled by averaged cooccurrence probabilities of word clusters.

Dagan, Marcus, \& Markovitch $(1993,1995)$ present a similarity-based model, which avoids building clusters. Instead, each word is modeled by its own specific class, a set of words that are most similar to it. Using this scheme, they predict which unobserved cooccurrences are more likely than others. Their model, however, does not provide probability estimates and so cannot be used as a component of a larger probabilistic model, as would be required in, say, speech recognition.

Class-based and similarity-based methods for cooccurrence modeling may at first sight seem to be special cases of clustering and weighted nearest-neighbor approaches used widely in machine learning and pattern recognition (Aha, Kibler, \& Albert, 1991; Cover \& Hart, 1967; Duda \& Hart, 1973; Stanfill \& Waltz, 1986; Devroye, Györfi, \& Lugosi, 1996; Atkeson, Moore, \& Schaal, 1997). There are important differences between those methods and ours. Clustering and nearest-neighbor techniques often rely on representing objects as points in a multidimensional space with coordinates determined by the values of intrinsic object features. However, in most language-modeling settings, all we know about a word are the frequencies of its cooccurrences with other words in certain configurations. Since the purpose of modeling is to estimate the probabilities of cooccurrences, the same cooccurrence statistics are the basis for both the similarity measure and the model predictions. That is, the only means we have for measuring word similarity are the predictions words make about what words they cooccur with, whereas in typical instance or (non-distributional) clustering 
learning methods, word similarity is defined from intrinsic features independently of the predictions (cooccurrence probabilities or classifications) associated with particular words (see for instance the work of Cardie (1993), $\mathrm{Ng} \&$ Lee (1996), Ng (1997), and Zavrel \& Daelemans (1997)).

\subsection{Main Contributions}

Our main contributions are a general scheme for using word similarity to improve the probability estimates of back-off models, and a comparative analysis of several similarity measures and parameter settings in two important language processing tasks, language modeling and disambiguation, showing that similarity-based estimates are indeed useful.

In our initial study, a language-model evaluation, we used a similarity-based model to estimate unseen bigram probabilities for Wall Street Journal text and compared it to a standard back-off model (Katz, 1987). Testing on a held-out sample, the similarity model achieved a $20 \%$ perplexity reduction over back-off for unseen bigrams. These constituted $10.6 \%$ of the test sample, leading to an overall reduction in test-set perplexity of $2.4 \%$. The similaritybased model was also tested in a speech-recognition task, where it yielded a statistically significant reduction (32 versus 64 mistakes in cases where there was disagreement with the back-off model) in recognition error.

In the disambiguation evaluation, we compared several variants of our initial method and the cooccurrence smoothing method of Essen \& Steinbiss (1992) against the estimation method of Katz in a decision task involving unseen pairs of direct objects and verbs. We found that all the similarity-based models performed almost $40 \%$ better than back-off, which yielded about $49 \%$ accuracy in our experimental setting. Furthermore, a scheme based on the Jensen-Shannon divergence (Rao, 1982; Lin, 1991) ${ }^{1}$ yielded statistically significant improvement in error rate over cooccurrence smoothing.

We also investigated the effect of removing extremely low-frequency events from the training set. We found that, in contrast to back-off smoothing, where such events are often discarded from training with little discernible effect, similarity-based smoothing methods suffer noticeable performance degradation when singletons (events that occur exactly once) are omitted.

The paper is organized as follows. Section 2 describes the general similarity-based framework; in particular, Section 2.3 presents the functions we use as measures of similarity. Section 3 details our initial language modeling experiments. Section 4 describes our comparison experiments on a pseudo-word disambiguation task. Section 5 discusses related work. Finally, Section 6 summarizes our contributions and outlines future directions.

\section{Distributional Similarity Models}

We wish to model conditional probability distributions arising from the cooccurrence of linguistic objects, typically words, in certain configurations. We thus consider pairs $\left(w_{1}, w_{2}\right) \in V_{1} \times V_{2}$ for appropriate sets $V_{1}$ and $V_{2}$, not necessarily disjoint. In what follows, we use subscript $i$ for the $i^{t h}$ element of a pair; thus $P\left(w_{2} \mid w_{1}\right)$ is the conditional probability (or rather, some empirical estimate drawn from a base language model, the true probability being unknown) that a pair has second element $w_{2}$ given that its first element is 
$w_{1}$; and $P\left(w_{1} \mid w_{2}\right)$ denotes the probability estimate, according to the base language model, that $w_{1}$ is the first word of a pair given that the second word is $w_{2} . P(w)$ denotes the base estimate for the unigram probability of word $w$.

A similarity-based language model consists of three parts: a scheme for deciding which word pairs require a similarity-based estimate, a method for combining information from similar words, and, of course, a function measuring the similarity between words. We give the details of each of these three parts in the following three sections. We will only be concerned with similarity between words in $V_{1}$, which are the conditioning events for the probabilities $P\left(w_{2} \mid w_{1}\right)$ that we want to estimate.

\subsection{Discounting and Redistribution}

Data sparseness makes the maximum likelihood estimate (MLE) for word pair probabilities unreliable. The MLE for the probability of a word pair $\left(w_{1}, w_{2}\right)$, conditional on the appearance of word $w_{1}$, is simply

$$
P_{M L}\left(w_{2} \mid w_{1}\right)=\frac{c\left(w_{1}, w_{2}\right)}{c\left(w_{1}\right)}
$$

where $c\left(w_{1}, w_{2}\right)$ is the frequency of $\left(w_{1}, w_{2}\right)$ in the training corpus and $c\left(w_{1}\right)$ is the frequency of $w_{1}$. However, $P_{M L}$ is zero for any unseen word pair, that is, any such pair would be predicted as impossible. More generally, the MLE is unreliable for events with small nonzero counts as well as for those with zero counts. In the language modeling literature, the term smoothing is used to refer to methods for adjusting the probability estimates of small-count events away from the MLE to try to alleviate its unreliability. Our proposals address the zero-count problem exclusively, and we rely on existing techniques to smooth other small counts.

Previous proposals for the zero-count problem (Good, 1953; Jelinek et al., 1992; Katz, 1987; Church \& Gale, 1991) adjust the MLE so that the total probability of seen word pairs is less than one, leaving some probability mass to be redistributed among the unseen pairs. In general, the adjustment involves either interpolation, in which the MLE is used in linear combination with an estimator guaranteed to be nonzero for unseen word pairs, or discounting, in which a reduced MLE is used for seen word pairs, with the probability mass left over from this reduction used to model unseen pairs.

The back-off method of Katz (1987) is a prime example of discounting:

$$
\hat{P}\left(w_{2} \mid w_{1}\right)=\left\{\begin{array}{ll}
P_{d}\left(w_{2} \mid w_{1}\right) & c\left(w_{1}, w_{2}\right)>0 \\
\alpha\left(w_{1}\right) P_{r}\left(w_{2} \mid w_{1}\right) & c\left(w_{1}, w_{2}\right)=0
\end{array},\right.
$$

where $P_{d}$ represents the Good-Turing discounted estimate (Katz, 1987) for seen word pairs, and $P_{r}$ denotes the model for probability redistribution among the unseen word pairs. $\alpha\left(w_{1}\right)$ is a normalization factor. Since an extensive comparison study by Chen \& Goodman (1996) indicated that back-off is better than interpolation for estimating bigram probabilities, we will not consider interpolation methods here; however, one could easily incorporate similarity-based estimates into an interpolation framework as well.

In his original back-off model, Katz used $P\left(w_{2}\right)$ as the model for predicting $\hat{P}\left(w_{2} \mid w_{1}\right)$ for unseen word pairs, that is, his model backed off to a unigram model for unseen bigrams. 
However, it is conceivable that backing off to a more detailed model than unigrams would be advantageous. Therefore, we generalize Katz's formulation by writing $P_{r}\left(w_{2} \mid w_{1}\right)$ instead of $P\left(w_{2}\right)$, enabling us to use similarity-based estimates for unseen word pairs instead of unigram frequency. Observe that similarity estimates are used for unseen word pairs only.

We next investigate estimates for $P_{r}\left(w_{2} \mid w_{1}\right)$ derived by averaging information from words that are distributionally similar to $w_{1}$.

\subsection{Combining Evidence}

Similarity-based models make the following assumption: if word $w_{1}^{\prime}$ is "similar" to word $w_{1}$, then $w_{1}^{\prime}$ can yield information about the probability of unseen word pairs involving $w_{1}$. We use a weighted average of the evidence provided by similar words, or neighbors, where the weight given to a particular word $w_{1}^{\prime}$ depends on its similarity to $w_{1}$.

More precisely, let $W\left(w_{1}, w_{1}^{\prime}\right)$ denote an increasing function of the similarity between $w_{1}$ and $w_{1}^{\prime}$, and let $\mathcal{S}\left(w_{1}\right)$ denote the set of words most similar to $w_{1}$. Then the general form of similarity model we consider is a $W$-weighted linear combination of predictions of similar words:

$$
P_{\mathrm{SIM}}\left(w_{2} \mid w_{1}\right)=\sum_{w_{1}^{\prime} \in \mathcal{S}\left(w_{1}\right)} \frac{W\left(w_{1}, w_{1}^{\prime}\right)}{\operatorname{norm}\left(w_{1}\right)} P\left(w_{2} \mid w_{1}^{\prime}\right)
$$

where $\operatorname{norm}\left(w_{1}\right)=\sum_{w_{1}^{\prime} \in \mathcal{S}\left(w_{1}\right)} W\left(w_{1}, w_{1}^{\prime}\right)$ is a normalization factor. According to this formula, $w_{2}$ is more likely to occur with $w_{1}$ if it tends to occur with the words that are most similar to $w_{1}$.

Considerable latitude is allowed in defining the set $\mathcal{S}\left(w_{1}\right)$, as is evidenced by previous work that can be put in the above form. Essen \& Steinbiss (1992) and Karov \& Edelman (1996) (implicitly) set $\mathcal{S}\left(w_{1}\right)=V_{1}$. However, it may be desirable to restrict $\mathcal{S}\left(w_{1}\right)$ in some fashion for efficiency reasons, especially if $V_{1}$ is large. For instance, in the language modeling application of Section 3, we use the closest $k$ or fewer words $w_{1}^{\prime}$ such that the dissimilarity between $w_{1}$ and $w_{1}^{\prime}$ is less than a threshold value $t ; k$ and $t$ are tuned experimentally.

One can directly replace $P_{r}\left(w_{2} \mid w_{1}\right)$ in the back-off equation (2) with $P_{\mathrm{SIM}}\left(w_{2} \mid w_{1}\right)$. However, other variations are possible, such as interpolating with the unigram probability $P\left(w_{2}\right)$ :

$$
P_{r}\left(w_{2} \mid w_{1}\right)=\gamma P\left(w_{2}\right)+(1-\gamma) P_{\text {SIM }}\left(w_{2} \mid w_{1}\right)
$$

This represents, in effect, a linear combination of the similarity estimate and the back-off estimate: if $\gamma=1$, then we have exactly Katz's back-off scheme. In the language modeling task (Section 3) we set $\gamma$ experimentally; to simplify our comparison of different similarity models for sense disambiguation (Section 4), we set $\gamma$ to 0 .

It would be possible to make $\gamma$ depend on $w_{1}$, so that the contribution of the similarity estimate could vary among words. Such dependences are often used in interpolated models (Jelinek \& Mercer, 1980; Jelinek et al., 1992; Saul \& Pereira, 1997) and are indeed advantageous. However, since they introduce hidden variables, they require a more complex training algorithm, and we did not pursue that direction in the present work. 


\subsection{Measures of Similarity}

We now consider several word similarity measures that can be derived automatically from the statistics of a training corpus, as opposed to being derived from manually-constructed word classes (Yarowsky, 1992; Resnik, 1992, 1995; Luk, 1995; Lin, 1997). Sections 2.3.1 and 2.3.2 discuss two related information-theoretic functions, the KL divergence and the Jensen-Shannon divergence. Section 2.3.3 describes the $L_{1}$ norm, a geometric distance function. Section 2.3.4 examines the confusion probability, which has been previously employed in language modeling tasks. There are, of course, many other possible functions; we have opted to restrict our attention to this reasonably diverse set.

For each function, a corresponding weight function $W\left(w_{1}, w_{1}^{\prime}\right)$ is given. The choice of weight function is to some extent arbitrary; the requirement that it be increasing in the similarity between $w_{1}$ and $w_{1}^{\prime}$ is not extremely constraining. While clearly performance depends on using a good weight function, it would be impossible to try all conceivable $W\left(w_{1}, w_{1}^{\prime}\right)$. Therefore, in section 4.5 , we describe experiments evaluating similarity-based models both with and without weight functions.

All the similarity functions we describe depend on some base language model $P\left(w_{2} \mid w_{1}\right)$, which may or may not be the Katz discounted model $\hat{P}\left(w_{2} \mid w_{1}\right)$ from Section 2.1 above. While we discuss the complexity of computing each similarity function, it should be noted that in our current implementation, this is a one-time cost: we construct the $\left|V_{1}\right| \times\left|V_{1}\right|$ matrix of word-to-word similarities before any parameter training takes place.

2.3.1. KL divergence The Kullback-Leibler $(K L)$ divergence is a standard informationtheoretic measure of the dissimilarity between two probability mass functions (Kullback, 1959; Cover \& Thomas, 1991). We can apply it to the conditional distributions induced by words in $V_{1}$ on words in $V_{2}$ :

$$
D\left(w_{1} \| w_{1}^{\prime}\right)=\sum_{w_{2}} P\left(w_{2} \mid w_{1}\right) \log \frac{P\left(w_{2} \mid w_{1}\right)}{P\left(w_{2} \mid w_{1}^{\prime}\right)}
$$

$D\left(w_{1} \| w_{1}^{\prime}\right)$ is non-negative, and is zero if and only if $P\left(w_{2} \mid w_{1}\right)=P\left(w_{2} \mid w_{1}^{\prime}\right)$ for all $w_{2}$. However, the KL divergence is non-symmetric and does not obey the triangle inequality.

For $D\left(w_{1} \| w_{1}^{\prime}\right)$ to be defined it must be the case that $P\left(w_{2} \mid w_{1}^{\prime}\right)>0$ whenever $P\left(w_{2} \mid w_{1}\right)>0$. Unfortunately, this generally does not hold for MLEs based on samples; we must use smoothed estimates that redistribute some probability mass to zero-frequency events. But this forces the sum in (4) to be over all $w_{2} \in V_{2}$, which makes this calculation expensive for large vocabularies.

Once the divergence $D\left(w_{1} \| w_{1}^{\prime}\right)$ is computed, we set

$$
W_{D}\left(w_{1}, w_{1}^{\prime}\right)=10^{-\beta D\left(w_{1} \| w_{1}^{\prime}\right)}
$$

The role of the free parameter $\beta$ is to control the relative influence of the neighbors closest to $w_{1}$ : if $\beta$ is high, then $W_{D}\left(w_{1}, w_{1}^{\prime}\right)$ is non-negligible only for those $w_{1}^{\prime}$ that are extremely close to $w_{1}$, whereas if $\beta$ is low, distant neighbors also contribute to the estimate. We chose a negative exponential function of the KL divergence for the weight function by analogy with 
the form of the cluster membership function in related distributional clustering work (Pereira et al., 1993) and also because that is the form for the probability that $w_{1}$ 's distribution arose from a sample drawn from the distribution of $w_{1}^{\prime}$ (Cover \& Thomas, 1991; Lee, 1997). However, these reasons are heuristic rather than theoretical, since we do not have a rigorous probabilistic justification for similarity-based methods.

\subsubsection{Jensen-Shannon divergence A related measure is the Jensen-Shannon divergence} (Rao, 1982; Lin, 1991), which can be defined as the average of the KL divergence of each of two distributions to their average distribution:

$$
J\left(w_{1}, w_{1}^{\prime}\right)=\frac{1}{2}\left[D\left(w_{1} \| \frac{w_{1}+w_{1}^{\prime}}{2}\right)+D\left(w_{1}^{\prime} \| \frac{w_{1}+w_{1}^{\prime}}{2}\right)\right],
$$

where $\left(w_{1}+w_{1}^{\prime}\right) / 2$ is shorthand for the distribution

$$
\frac{1}{2}\left(P\left(w_{2} \mid w_{1}\right)+P\left(w_{2} \mid w_{1}^{\prime}\right)\right)
$$

Since the KL divergence is nonnegative, $J\left(w_{1}, w_{1}^{\prime}\right)$ is also nonnegative. Furthermore, letting $p\left(w_{2}\right)=P\left(w_{2} \mid w_{1}\right)$ and $p^{\prime}\left(w_{2}\right)=P\left(w_{2} \mid w_{1}^{\prime}\right)$, it is easy to see that

$$
J\left(w_{1}, w_{1}^{\prime}\right)=H\left(\frac{p+p^{\prime}}{2}\right)-\frac{1}{2} H(p)-\frac{1}{2} H\left(p^{\prime}\right) \quad,
$$

where $H(q)=-\sum_{w} q(w) \log q(w)$ is the entropy of the discrete density $q$. This equation shows that $J$ gives the information gain achieved by distinguishing the two distributions $p$ and $p^{\prime}$ (conditioning on contexts $w_{1}$ and $w_{1}^{\prime}$ ) over pooling the two distributions (ignoring the distinction between $w_{1}$ and $w_{1}^{\prime}$ ).

It is also easy to see that $J$ can be computed efficiently, since it depends only on those conditioned words that occur in both contexts. Indeed, letting $\mathcal{C}=\left\{w_{2}: p\left(w_{2}\right)>\right.$ $\left.0, p^{\prime}\left(w_{2}\right)>0\right\}$, and grouping the terms of (6) appropriately, we obtain

$$
J\left(w_{1}, w_{1}^{\prime}\right)=\log 2+\frac{1}{2} \sum_{w_{2} \in \mathcal{C}}\left\{h\left(p\left(w_{2}\right)+p^{\prime}\left(w_{2}\right)\right)-h\left(p\left(w_{2}\right)\right)-h\left(p^{\prime}\left(w_{2}\right)\right)\right\}
$$

where $h(x)=-x \log x$. Therefore, $J\left(w_{1}, w_{1}^{\prime}\right)$ is bounded, ranging between 0 and $\log 2$, and smoothed estimates are not required because probability ratios are not involved.

As in the KL divergence case, we set $W_{J}\left(w_{1}, w_{1}^{\prime}\right)=10^{-\beta J\left(w_{1}, w_{1}^{\prime}\right)} ; \beta$ plays the same role as before.

2.3.3. $L_{1}$ norm The $L_{1}$ norm is defined as

$$
L\left(w_{1}, w_{1}^{\prime}\right)=\sum_{w_{2}}\left|P\left(w_{2} \mid w_{1}\right)-P\left(w_{2} \mid w_{1}^{\prime}\right)\right| \quad .
$$

By grouping terms as before, we can express $L\left(w_{1}, w_{1}^{\prime}\right)$ in a form depending only on the "common" $w_{2}$ : 


$$
L\left(w_{1}, w_{1}^{\prime}\right)=2-\sum_{w_{2} \in \mathcal{C}} p\left(w_{2}\right)-\sum_{w_{2} \in \mathcal{C}} p^{\prime}\left(w_{2}\right)+\sum_{w_{2} \in \mathcal{C}}\left|p\left(w_{2}\right)-p^{\prime}\left(w_{2}\right)\right|
$$

It follows from the triangle inequality that $0 \leq L\left(w_{1}, w_{1}^{\prime}\right) \leq 2$, with equality to 0 if and only if there are no words $w_{2}$ such that both $P\left(w_{2} \mid w_{1}\right)$ and $P\left(w_{2} \mid w_{1}^{\prime}\right)$ are strictly positive.

Since we require a weighting scheme that is decreasing in $L$, we set

$$
W_{L}\left(w_{1}, w_{1}^{\prime}\right)=\left(2-L\left(w_{1}, w_{1}^{\prime}\right)\right)^{\beta} \quad,
$$

with $\beta$ again free. ${ }^{2}$ As before, the higher $\beta$ is, the more relative influence is accorded to the nearest neighbors.

It is interesting to note the following relations between the $L_{1}$ norm, the KL-divergence, and the Jensen-Shannon divergence. Cover \& Thomas (1991) give the following lower bound:

$$
L\left(w_{1}, w_{1}^{\prime}\right) \leq \sqrt{D\left(w_{1} \| w_{1}^{\prime}\right) \cdot 2 \log b}
$$

where $b$ is the base of the logarithm function. Lin (1991) notes that $L$ is an upper bound for $J$ :

$$
J\left(w_{1}, w_{1}^{\prime}\right) \leq L\left(w_{1}, w_{1}^{\prime}\right)
$$

2.3.4. Confusion probability Extending work by Sugawara, Nishimura, Toshioka, Okochi, \& Kaneko (1985), Essen \& Steinbiss (1992) used confusion probability to estimate word cooccurrence probabilities. ${ }^{3}$ They report $14 \%$ improvement in test-set perplexity (defined below) on a small corpus. The confusion probability was also used by Grishman \& Sterling (1993) to estimate the likelihood of selectional patterns.

The confusion probability is an estimate of the probability that word $w_{1}^{\prime}$ can be substituted for word $w_{1}$, in the sense of being found in the same contexts:

$$
\begin{aligned}
P_{\mathrm{C}}\left(w_{1}^{\prime} \mid w_{1}\right) & =\sum_{w_{2}} \frac{P\left(w_{1} \mid w_{2}\right) P\left(w_{1}^{\prime} \mid w_{2}\right) P\left(w_{2}\right)}{P\left(w_{1}\right)} \\
& =W_{\mathrm{C}}\left(w_{1}, w_{1}^{\prime}\right)
\end{aligned}
$$

$\left(P\left(w_{1}\right)\right.$ serves as a normalization factor). In contrast to the distance functions described above, $P_{\mathrm{C}}$ has the curious property that $w_{1}$ may not necessarily be the "closest" word to itself, that is, there may exist a word $w_{1}^{\prime}$ such that $P_{\mathrm{C}}\left(w_{1}^{\prime} \mid w_{1}\right)>P_{\mathrm{C}}\left(w_{1} \mid w_{1}\right)$; see Section 4.4 for an example.

The confusion probability can be computed from empirical estimates provided all unigram estimates are nonzero (as we assume throughout). In fact, the use of smoothed estimates such as those provided by Katz's back-off scheme is problematic, because those estimates typically do not preserve consistency with respect to marginal estimates and Bayes's rule (that is, it may be that $\sum_{w_{2}} P\left(w_{1} \mid w_{2}\right) P\left(w_{2}\right) \neq P\left(w_{1}\right)$ ). However, using consistent estimates (such as the MLE), we can safely apply Bayes's rule to rewrite $P_{\mathrm{C}}$ as follows:

$$
P_{\mathrm{C}}\left(w_{1}^{\prime} \mid w_{1}\right)=\sum_{w_{2}} \frac{P\left(w_{2} \mid w_{1}\right)}{P\left(w_{2}\right)} \cdot P\left(w_{2} \mid w_{1}^{\prime}\right) P\left(w_{1}^{\prime}\right)
$$


Table 1. Summary of Similarity Function Properties

\begin{tabular}{l|l|c|c} 
name & range & base LM constraints & tune? \\
\hline$D$ & {$[0, \infty]$} & $P\left(w_{2} \mid w_{1}^{\prime}\right) \neq 0$ if $P\left(w_{2} \mid w_{1}\right) \neq 0$ & yes \\
$J$ & {$[0, \log 2]$} & none & yes \\
$L$ & {$[0,2]$} & none & yes \\
$P_{\mathrm{C}}$ & {$\left[0, \frac{1}{2} \max _{w_{2}} P\left(w_{2}\right)\right]$} & Bayes consistency & no
\end{tabular}

As with the Jensen-Shannon divergence and the $L_{1}$ norm, this sum requires computation only over the "common" $w_{2}$ 's.

Examination of Equation (8) reveals an important difference between the confusion probability and the functions $D, J$, and $L$ described in the previous sections. Those functions rate $w_{1}^{\prime}$ as similar to $w_{1}$ if, roughly, $P\left(w_{2} \mid w_{1}^{\prime}\right)$ is high when $P\left(w_{2} \mid w_{1}\right)$ is. $P_{\mathrm{C}}\left(w_{1}^{\prime} \mid w_{1}\right)$, however, is greater for those $w_{1}^{\prime}$ for which $P\left(w_{1}^{\prime}, w_{2}\right)$ is large when $P\left(w_{2} \mid w_{1}\right) / P\left(w_{2}\right)$ is. When this ratio is large, we may think of $w_{2}$ as being exceptional, since if $w_{2}$ is infrequent, we do not expect $P\left(w_{2} \mid w_{1}\right)$ to be large.

2.3.5. Summary Several features of the measures of similarity listed above are summarized in Table 1. "Base LM constraints" are conditions that must be satisfied by the probability estimates of the base language model. The last column indicates whether the weight $W\left(w_{1}, w_{1}^{\prime}\right)$ associated with each similarity function depends on a parameter that needs to be tuned experimentally.

\section{Language Modeling}

The goal of our first set of experiments, described in this section, was to provide proof of concept by showing that similarity-based models can achieve better language modeling performance than back-off. We therefore only used one similarity measure. The success of these experiments convinced us that similarity-based methods are worth examining more closely; the results of our second set of experiments, comparing several similarity functions on a pseudo-word disambiguation task, are described in the next section.

Our language modeling experiments used a similarity-based model, with the KL divergence as (dis)similarity measure, as an alternative to unigram frequency when backing off in a bigram model. That is, we used the bigram language model defined by:

$$
\begin{aligned}
\hat{P}\left(w_{2} \mid w_{1}\right) & = \begin{cases}P_{d}\left(w_{2} \mid w_{1}\right) & c\left(w_{1}, w_{2}\right)>0 \\
\alpha\left(w_{1}\right) P_{r}\left(w_{2} \mid w_{1}\right) & c\left(w_{1}, w_{2}\right)=0\end{cases} \\
P_{r}\left(w_{2} \mid w_{1}\right) & =\gamma P\left(w_{2}\right)+(1-\gamma) P_{\mathrm{SIM}}\left(w_{2} \mid w_{1}\right) \\
P_{\mathrm{SIM}}\left(w_{2} \mid w_{1}\right) & =\sum_{w_{1}^{\prime} \in \mathcal{S}\left(w_{1}\right)} \frac{W\left(w_{1}, w_{1}^{\prime}\right)}{\operatorname{norm}\left(w_{1}\right)} P\left(w_{2} \mid w_{1}^{\prime}\right) \\
W\left(w_{1}, w_{1}^{\prime}\right) & =10^{-\beta D\left(w_{1}|| w_{1}^{\prime}\right)},
\end{aligned}
$$


Table 2. Perplexity Reduction on Unseen Bigrams for Different Model Parameters

\begin{tabular}{rlllll}
\multicolumn{1}{c}{$k$} & $t$ & $\beta$ & $\gamma$ & training reduction $(\%)$ & test reduction $(\%)$ \\
\hline 60 & 2.5 & 4.0 & 0.15 & 18.4 & 20.51 \\
50 & 2.5 & 4.0 & 0.15 & 18.38 & 20.45 \\
40 & 2.5 & 4.0 & 0.2 & 18.34 & 20.03 \\
30 & 2.5 & 4.0 & 0.25 & 18.33 & 19.76 \\
70 & 2.5 & 4.0 & 0.1 & 18.3 & 20.53 \\
80 & 2.5 & 4.5 & 0.1 & 18.25 & 20.55 \\
100 & 2.5 & 4.5 & 0.1 & 18.23 & 20.54 \\
90 & 2.5 & 4.5 & 0.1 & 18.23 & 20.59 \\
20 & 1.5 & 4.0 & 0.3 & 18.04 & 18.7 \\
10 & 1.5 & 3.5 & 0.3 & 16.64 & 16.94
\end{tabular}

where $V_{1}=V_{2}=V$, the entire vocabulary. As noted earlier, the estimates of $P\left(w_{2} \mid w_{1}^{\prime}\right)$ must be smoothed to avoid division by zero when computing $D\left(w_{1} \| w_{1}^{\prime}\right)$; we employed the standard Katz bigram back-off model for that purpose. Since $|V|=20,000$ in this application, we considered only a small fraction of $V$ in computing $P_{\text {SIM }}$, using the tunable thresholds $k$ and $t$ described in Section 2.2 for this purpose.

The standard evaluation metric for language models is the likelihood of the test data according to the model, or, more intuitively, the test-set perplexity

$$
\sqrt[N]{\prod_{i=1}^{N} P\left(w_{i} \mid w_{i-1}\right)^{-1}}
$$

which represents the average number of alternatives presented by the (bigram) model after each test word. Thus, a better model will have a lower perplexity. In our task, lower perplexity will indicate better prediction of unseen bigrams.

We evaluated the above model by comparing its test-set perplexity and effect on speechrecognition accuracy with the baseline bigram back-off model developed by MIT Lincoln Laboratories for the Wall Street Journal (WSJ) text and dictation corpora provided by ARPA's HLT program (Paul, 1991). ${ }^{4}$ The baseline back-off model follows the Katz design, except that, for the sake of compactness, all frequency one bigrams are ignored. The counts used in this model and in ours were obtained from 40.5 million words of WSJ text from the years 1987-89.

For perplexity evaluation, we tuned the similarity model parameters by minimizing perplexity on an additional sample of 57.5 thousand words of WSJ text, drawn from the ARPA HLT development test set. The best parameter values found were $k=60, t=2.5, \beta=4$ and $\gamma=0.15$. For these values, the improvement in perplexity for unseen bigrams in a held-out 18 thousand word sample (the ARPA HLT evaluation test set) is just over $20 \%$. Since unseen bigrams comprise $10.6 \%$ of this sample, the improvement on unseen bigrams corresponds to an overall test set perplexity improvement of $2.4 \%$ (from 237.4 to 231.7 ). Table 2 shows reductions in training and test perplexity, sorted by training reduction, for different choices of the number $k$ of closest neighbors used. The values of $\beta, \gamma$ and $t$ are the best ones found for each $k^{5}$ 
From equation (9), it is clear that the computational cost of applying the similarity model to an unseen bigram is $O(k)$. Therefore, lower values for $k$ (and $t$ ) are computationally preferable. From the table, we can see that reducing $k$ to 30 incurs a penalty of less than $1 \%$ in the perplexity improvement, so relatively low values of $k$ appear to be sufficient to achieve most of the benefit of the similarity model. As the table also shows, the best value of $\gamma$ increases as $k$ decreases; that is, for lower $k$, a greater weight is given to the conditioned word's frequency. This suggests that the predictive power of neighbors beyond the closest 30 or so can be modeled fairly well by the overall frequency of the conditioned word.

The bigram similarity model was also tested as a language model in speech recognition. The test data for this experiment were pruned word lattices for 403 WSJ closed-vocabulary test sentences. Arc scores in these lattices are sums of an acoustic score (negative log likelihood) and a language-model score, which in this case was the negative log probability provided by the baseline bigram model.

From the given lattices, we constructed new lattices in which the arc scores were modified to use the similarity model instead of the baseline model. We compared the best sentence hypothesis in each original lattice with the best hypothesis in the modified one, and counted the word disagreements in which one of the hypotheses was correct. There were a total of 96 such disagreements; the similarity model was correct in 64 cases, and the back-off model in 32. This advantage for the similarity model is statistically significant at the 0.01 level. The overall reduction in error rate is small, from $21.4 \%$ to $20.9 \%$, because the number of disagreements is small compared with the overall number of errors in the recognition setup employed in these experiments.

Table 3 shows some examples of speech recognition disagreements between the two models. The hypotheses are labeled ' $\mathrm{B}$ ' for back-off and ' $\mathrm{S}$ ' for similarity, and the boldface words are errors. The similarity model seems to be better at modeling regularities such as semantic parallelism in lists and avoiding a past tense form after "to." On the other hand, the similarity model makes several mistakes in which a function word is inserted in a place where punctuation would be found in written text.

\section{Word-Sense Disambiguation}

Since the experiments described in the previous section demonstrated promising results for similarity-based estimation, we ran a second set of experiments designed to help us compare and analyze the somewhat diverse set of similarity measures given in Table 1. Unfortunately, the KL divergence and the confusion probability have different requirements on the base language model, and so we could not run a direct four-way comparison. As explained below, we elected to omit the KL divergence from consideration.

We chose to evaluate the three remaining measures on a word sense disambiguation task, in which each method was presented with a noun and two verbs, and was asked which verb was more likely to have the noun as a direct object. Thus, we did not measure the absolute quality of the assignment of probabilities, as would be the case in a perplexity evaluation, but rather the relative quality. We could therefore ignore constant factors, which is why we did not normalize the similarity measures. 
Table 3. Speech Recognition Disagreements between Models

\begin{tabular}{l|l} 
B $\mid$ commitments . . f from leaders felt the three point six billion dollars \\
\hline $\mathrm{S} \mid$ commitments . . f from leaders fell to three point six billion dollars \\
\hline $\mathrm{B} \mid$ followed by France the US agreed in Italy \\
\hline $\mathrm{S} \mid$ followed by France the US Greece ... Italy \\
\hline $\mathrm{B} \mid$ he whispers to made a \\
\hline $\mathrm{S} \mid$ he whispers to an aide \\
\hline $\mathrm{B} \mid$ the necessity for change exist \\
\hline $\mathrm{S} \mid$ the necessity for change exists \\
\hline \\
\hline $\mathrm{B} \mid$ without ... additional reserves Centrust would have reported \\
\hline $\mathrm{S} \mid$ without ... additional reserves of Centrust would have reported \\
\hline $\mathrm{B} \mid$ in the darkness past the church \\
\hline $\mathrm{S} \mid$ in the darkness passed the church
\end{tabular}

\subsection{Task Definition}

In the usual word sense disambiguation problem, the method to be tested is presented with an ambiguous word in some context, and is asked to identify the correct sense of the word from that context. For example, a test instance might be the sentence fragment "robbed the bank"; the question is whether "bank" refers to a river bank, a savings bank, or perhaps some other alternative meaning.

While sense disambiguation is clearly an important problem for language processing applications, as an evaluation task it presents numerous experimental difficulties. First, the very notion of "sense" is not clearly defined; for instance, dictionaries may provide sense distinctions that are too fine or too coarse for the data at hand. Also, one needs to have training data for which the correct senses have been assigned; acquiring these correct senses generally requires considerable human effort. Furthermore, some words have many possible senses, whereas others are essentially monosemous; this means that test cases are not all uniformly hard.

To circumvent these and other difficulties, we set up a pseudo-word disambiguation experiment (Schütze, 1992a; Gale, Church, \& Yarowsky, 1992), the format of which is as follows. First, a list of pseudo-words is constructed, each of which is the combination of two different words in $V_{2}$. Each word in $V_{2}$ contributes to exactly one pseudo-word. Then, every $w_{2}$ in the test set is replaced with its corresponding pseudo-word. For example, if a pseudo-word is created out of the words "make" and "take", then the data is altered as follows: 


$$
\begin{array}{ll}
\text { make } & \text { plans } \Rightarrow\{\text { make, take }\} \text { plans } \\
\text { take } & \text { action } \Rightarrow\{\text { make, take }\} \text { action }
\end{array}
$$

The method being tested must choose between the two words that make up the pseudo-word.

The advantages of using pseudo-words are two-fold. First, the alternative "senses" are under the control of the experimenter. Each test instance presents exactly two alternatives to the disambiguation method, and the alternatives can be chosen to be of the same frequency, the same part of speech, and so on. Secondly, the pre-transformation data yields the correct answer, so that no hand-tagging of the word senses is necessary. These advantages make pseudo-word experiments an elegant and simple means to test the efficacy of different language models; of course they may not provide a completely accurate picture of how the models would perform in real disambiguation tasks, although one could create more realistic settings by making pseudo-words out of more than two words, varying the frequencies of the alternative pseudo-senses, and so on.

For ease of comparison, we did not consider interpolation with unigram probabilities. Thus, the model we used for these experiments differs slightly from that used in the language modeling tests; it can be summarized as follows:

$$
\begin{aligned}
\hat{P}\left(w_{2} \mid w_{1}\right) & = \begin{cases}P_{d}\left(w_{2} \mid w_{1}\right) & c\left(w_{1}, w_{2}\right)>0 \\
\alpha\left(w_{1}\right) P_{r}\left(w_{2} \mid w_{1}\right) & c\left(w_{1}, w_{2}\right)=0\end{cases} \\
P_{r}\left(w_{2} \mid w_{1}\right) & =P_{\mathrm{SIM}}\left(w_{2} \mid w_{1}\right) \\
P_{\mathrm{SIM}}\left(w_{2} \mid w_{1}\right) & =\sum_{w_{1}^{\prime} \in \mathcal{S}\left(w_{1}\right)} \frac{W\left(w_{1}, w_{1}^{\prime}\right)}{\operatorname{norm}\left(w_{1}\right)} P\left(w_{2} \mid w_{1}^{\prime}\right)
\end{aligned}
$$

\subsection{Data}

We used a statistical part-of-speech tagger (Church, 1988) and pattern matching and concordancing tools (due to David Yarowsky) to identify transitive main verbs $\left(V_{2}\right)$ and head nouns $\left(V_{1}\right)$ of the corresponding direct objects in 44 million words of 1988 Associated Press newswire. We selected the noun-verb pairs for the 1000 most frequent nouns in the corpus. These pairs are undoubtedly somewhat noisy given the errors inherent in the part-of-speech tagging and pattern matching.

We used $80 \%$, or 587,833 , of the pairs so derived for building models, reserving $20 \%$ for testing purposes. As some, but not all, of the similarity measures require smoothed models, we calculated both a Katz back-off model $\left(P=\hat{P}\right.$ in equation (2), with $P_{r}\left(w_{2} \mid w_{1}\right)=$ $P\left(w_{2}\right)$ ), and a maximum-likelihood model $\left(P=P_{\mathrm{ML}}\right)$. Furthermore, we wished to evaluate the hypothesis that a more compact language model can be built without affecting model quality by deleting singletons, word pairs that occur only once, from the training set. This claim had been made in particular for language modeling (Katz, 1987). We therefore built four base models, summarized in Table 4 .

Since we wished to test the effectiveness of using similarity for unseen word cooccurrences, we removed from the test data any verb-object pairs that occurred in the training set; this resulted in 17, 152 unseen pairs (some occurred multiple times). The unseen pairs 
Table 4. Base Language Models

\begin{tabular}{c|cc} 
& $\begin{array}{c}\text { with singletons } \\
(587,833 \text { pairs })\end{array}$ & $\begin{array}{c}\text { no singletons } \\
(505,426 \text { pairs })\end{array}$ \\
\hline MLE & MLE-1 & MLE-o1 \\
Katz & BO-1 & BO-o1
\end{tabular}

were further divided into five equal-sized parts, $T_{1}$ through $T_{5}$, which formed the basis for fivefold cross-validation: in each of five runs, one of the $T_{i}$ was used as a performance test set, with the other four combined into one set used for tuning parameters (if necessary) via a simple grid search that evaluated the error on the tuning set at regularly spaced points in parameter space. Finally, test pseudo-words were created from pairs of verbs with similar frequencies, so as to control for word frequency in the decision task. Our method was to simply rank the verbs by frequency and create pseudo-words out of all adjacent pairs (thus, each verb participated in exactly one pseudoword). Table 5 lists some randomly chosen pseudowords and the frequencies of the corresponding verbs.

Table 5. Sample pseudoword verbs and frequencies. The word "meeet" is a typo occurring in the corpus.

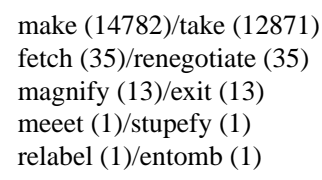

We use error rate as our performance metric, defined as

$$
\frac{1}{N}(\# \text { of incorrect choices }+(\# \text { of ties }) / 2)
$$

where $N$ was the size of the test corpus. A tie occurs when the two words making up a pseudo-word are deemed equally likely.

\subsection{Baseline Experiments}

The performances of the four base language models are shown in Table 6. MLE-1 and MLE-o1 both have error rates of exactly .5 because the test sets consist of unseen bigrams, which are all assigned a probability of 0 by maximum-likelihood estimates, and thus are all ties for this method. The back-off models BO-1 and BO-o1 also perform similarly.

Since the back-off models consistently performed worse than the MLE models, we chose to use only the MLE models in our subsequent experiments. Therefore, we only ran comparisons between the measures that could utilize unsmoothed data, namely, the $L_{1}$ norm, $L\left(w_{1}, w_{1}^{\prime}\right)$; the Jensen-Shannon divergence, $J\left(w_{1}, w_{1}^{\prime}\right)$; and the confusion probability, $P_{\mathrm{C}}\left(w_{1}^{\prime} \mid w_{1}\right) .{ }^{6}$ 
Table 6. Base Language Model Error Rates

\begin{tabular}{l|lllll} 
& $T_{1}$ & $T_{2}$ & $T_{3}$ & $T_{4}$ & $T_{5}$ \\
\hline MLE-1 & .5 & .5 & .5 & .5 & .5 \\
MLE-o1 & $"$ & " & " & " & " \\
BO-1 & 0.517 & 0.520 & 0.512 & 0.513 & 0.516 \\
BO-o1 & 0.517 & 0.520 & 0.512 & 0.513 & 0.516
\end{tabular}

Table 7. 10 closest words to the word "guy" for $L, J$, and $P_{\mathrm{C}}$, using MLE-1 as the base language model. The rank of the words "role" and "kid" are also shown if they are not among the top ten.

\begin{tabular}{ll|ll|ll}
\multicolumn{2}{c}{$L$} & \multicolumn{2}{c}{$P_{\mathrm{C}}$} \\
\hline GUY & 0.0 & GUY & 0.0 & role & 0.033 \\
kid & 1.23 & kid & 0.15 & people & 0.024 \\
lot & 1.35 & thing & 0.1645 & fire & 0.013 \\
thing & 1.39 & lot & 0.165 & GUY & 0.0127 \\
man & 1.46 & man & 0.175 & man & 0.012 \\
doctor & 1.46 & mother & 0.184 & year & 0.01 \\
girl & 1.48 & doctor & 0.185 & lot & 0.0095 \\
rest & 1.485 & friend & 0.186 & today & 0.009 \\
son & 1.497 & boy & 0.187 & way & 0.008778 \\
bit & 1.498 & son & 0.188 & part & 0.008772 \\
(role: rank 173) & \multicolumn{2}{l}{ (role: rank 43) } & (kid: rank 80)
\end{tabular}

\subsection{Sample Closest Words}

In this section, we examine the closest words to a randomly selected noun, "guy", according to the three measures $L, J$, and $P_{\mathrm{C}}$.

Table 7 shows the ten closest words, in order, when the base language model is MLE-1. There is some overlap between the closest words for $L$ and the closest words for $J$, but very little overlap between the closest words for these measures and the closest words with respect to $P_{\mathrm{C}}$ : only the words "man" and "lot" are common to all three. Also observe that the word "guy" itself is only fourth on the list of words with the highest confusion probability with respect to "guy".

Let us examine the case of the nouns "kid" and "role" more closely. According to the similarity functions $L$ and $J$, "kid" is the second closest word to "guy", and "role" is considered relatively distant. In the $P_{\mathrm{C}}$ case, however, "role" has the highest confusion probability with respect to "guy," whereas "kid" has only the 80th highest confusion probability. What accounts for these differences?

Table 8, which gives the ten verbs most likely to occur with "guy", "kid", and "role", indicates that both $L$ and $J$ rate words as similar if they tend to cooccur with the same verbs. Observe that four of the ten most likely verbs to occur with "kid" are also very likely 
Table 8. For each noun $w_{1}$, the ten verbs $w_{2}$ with highest $P\left(w_{2} \mid w_{1}\right)$. Bold-face verbs occur with both the given noun and with "guy." The base language model is MLE-1.

\begin{tabular}{l|l} 
Noun & Most Likely Verbs \\
\hline guy & see get play let give catch tell do pick need \\
\hline $\begin{array}{l}\text { kid } \\
\text { role }\end{array}$ & $\begin{array}{l}\text { get see take help want tell teach send give love } \\
\text { play take lead support assume star expand accept sing limit }\end{array}$
\end{tabular}

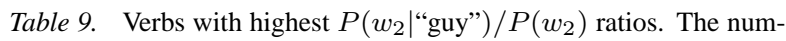
bers in parentheses are ranks.

(1) electrocute (2) shortchange (3) bedevil (4) admire (5) bore (6) fool (7) bless $\cdots(26)$ play $\cdots$ (49) get $\cdots$

Table 10. 10 closest words to the word "guy" for $L, J$, and $P_{\mathrm{C}}$, using MLE-o1 as the base language model.

\begin{tabular}{ll|ll|ll}
\multicolumn{2}{c}{$L$} & \multicolumn{2}{c}{$J$} & \multicolumn{2}{c}{$P_{\mathrm{C}}$} \\
\hline GUY & 0.0 & GUY & 0.0 & role & 0.05 \\
kid & 1.17 & kid & 0.15 & people & 0.025 \\
lot & 1.40 & thing & 0.16 & fire & 0.021 \\
thing & 1.41 & lot & 0.17 & GUY & 0.018 \\
reason & 1.417 & mother & 0.182 & work & 0.016 \\
break & 1.42 & answer & 0.1832 & man & 0.012 \\
ball & 1.439 & reason & 0.1836 & lot & 0.0113 \\
answer & 1.44 & doctor & 0.187 & job & 0.01099 \\
tape & 1.449 & boost & 0.189 & thing & 0.01092 \\
rest & 1.453 & ball & 0.19 & reporter & 0.0106
\end{tabular}

to occur with "guy", whereas only the verb "play" commonly occurs with both "role" and "guy".

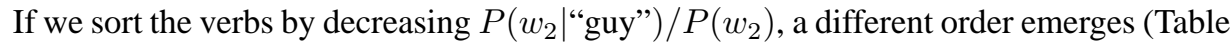
9): "play", the most likely verb to cooccur with "role", is ranked higher than "get", the most likely verb to cooccur with "kid", thus indicating why "role" has a higher confusion probability with respect to "guy" than "kid" does.

Finally, we examine the effect of deleting singletons from the base language model. Table 10 shows the ten closest words, in order, when the base language model is MLE-o1. The relative order of the four closest words remains the same; however, the next six words are quite different from those for MLE-1. This data suggests that the effect of singletons on calculations of similarity is quite strong, as is borne out by the experimental evaluations described in Section 4.5. 
We conjecture that this effect is due to the fact that there are many very low-frequency verbs in the data ( $65 \%$ of the verbs appeared with 10 or fewer nouns; the most common verb occurred with 710 nouns). Omitting singletons involving such verbs may well drastically alter the number of verbs that cooccur with both of two given nouns $w_{1}$ and $w_{1}^{\prime}$. Since the similarity functions we consider in this set of experiments depend on such words, it is not surprising that the effect of deleting singletons is rather dramatic. In contrast, a backoff language model is not as sensitive to missing singletons because of the Good-Turing discounting of small counts and inflation of zero counts.

\subsection{Performance of Similarity-Based Methods}

Figure 1 shows the results of our experiments on the five test sets, using MLE-1 as the base language model. The parameter $\beta$ was always set to the optimal value for the corresponding training set. RAND, which is shown for comparison purposes, simply chooses the weights $W\left(w_{1}, w_{1}^{\prime}\right)$ randomly. $\mathcal{S}\left(w_{1}\right)$ was set equal to $V_{1}$ in all cases.

The similarity-based methods consistently outperformed Katz's back-off method and the MLE (recall that both yielded error rates of about .5) by a large margin, indicating that information from other word pairs is very useful for unseen pairs when unigram frequency is not informative. The similarity-based methods also do much better than RAND, which indicates that it is not enough to simply combine information from other words arbitrarily: word similarity should be taken into account. In all cases, $J$ edged out the other methods. The average improvement in using $J$ instead of $P_{\mathrm{C}}$ is .0082 ; this difference is significant to the .1 level ( $p<.085)$, according to the paired t-test.

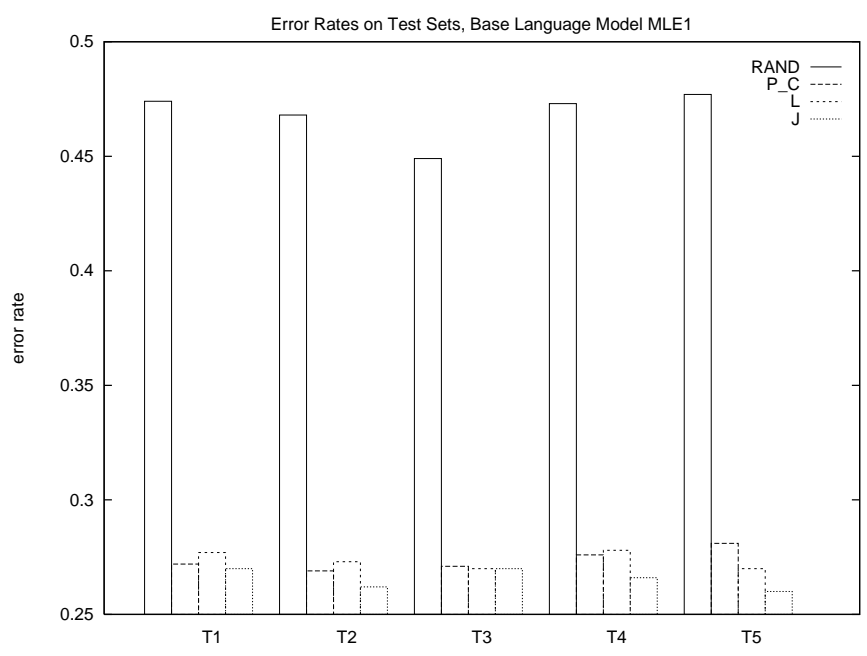

Figure 1. Error rates for each test set, where the base language model was MLE-1. The methods, going from left to right, are RAND , $P_{\mathrm{C}}, L$, and $J$. The performances shown are for settings of $\beta$ that were optimal for the corresponding training set. $\beta$ ranged from 4.0 to 4.5 for $L$ and from 20 to 26 for $J$. 
The results for the MLE-o1 case are depicted in Figure 2. Again, we see the similaritybased methods achieving far lower error rates than the MLE, back-off, and RAND methods, and again, $J$ always performed the best. However, omitting singletons amplified the disparity between $J$ and $P_{\mathrm{C}}$ : the average difference was .024 , which is significant to the .01 level (paired t-test).

An important observation is that all methods, including RAND, suffered a performance hit if singletons were deleted from the base language model. This seems to indicate that seen bigrams should be treated differently from unseen bigrams, even if the seen bigrams are extremely rare. We thus conclude that one cannot create a compressed similarity-based language model by omitting singletons without hurting performance, at least for this task.

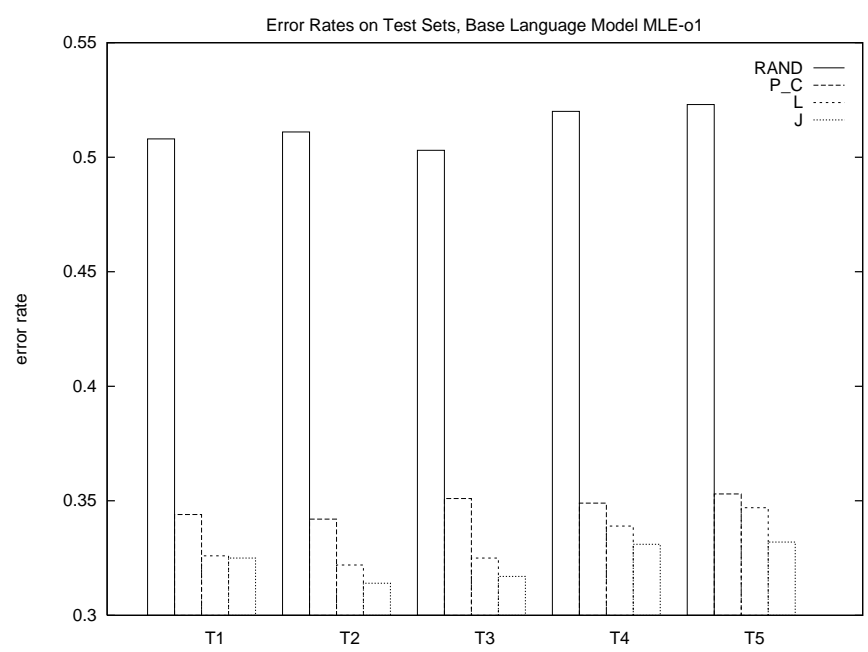

Figure 2. Error rates for each test set, where the base language model was MLE-o1. $\beta$ ranged from 6 to 11 for $L$ and from 21 to 22 for $J$.

We now analyze the role of the parameter $\beta$. Recall that $\beta$ appears in the weight functions for the Jensen-Shannon divergence and the $L_{1}$ norm:

$$
W_{J}\left(w_{1}, w_{1}^{\prime}\right)=10^{-\beta J\left(w_{1}, w_{1}^{\prime}\right)}, W_{L}\left(w_{1}, w_{1}^{\prime}\right)=\left(2-L\left(w_{1}, w_{1}^{\prime}\right)\right)^{\beta} \quad .
$$

It controls the relative influence of the most similar words: their influence increases with higher values of $\beta$.

Figure 3 shows how the value of $\beta$ affects disambiguation performance. Four curves are shown, each corresponding to a choice of similarity function and base language model. The error bars depict the average and range of error rates over the five disjoint test sets.

It is immediately clear that to get good performance results, $\beta$ must be set much higher for the Jensen-Shannon divergence than for the $L_{1}$ norm. This phenomenon results from the fact that the range of possible values for $J$ is much smaller than that for $L$. This "compression" of $J$ values requires a large $\beta$ to scale differences of distances correctly.

We also observe that setting $\beta$ too low causes substantially worse error rates; however, the curves level off rather than moving upwards again. That is, as long as a sufficiently large 


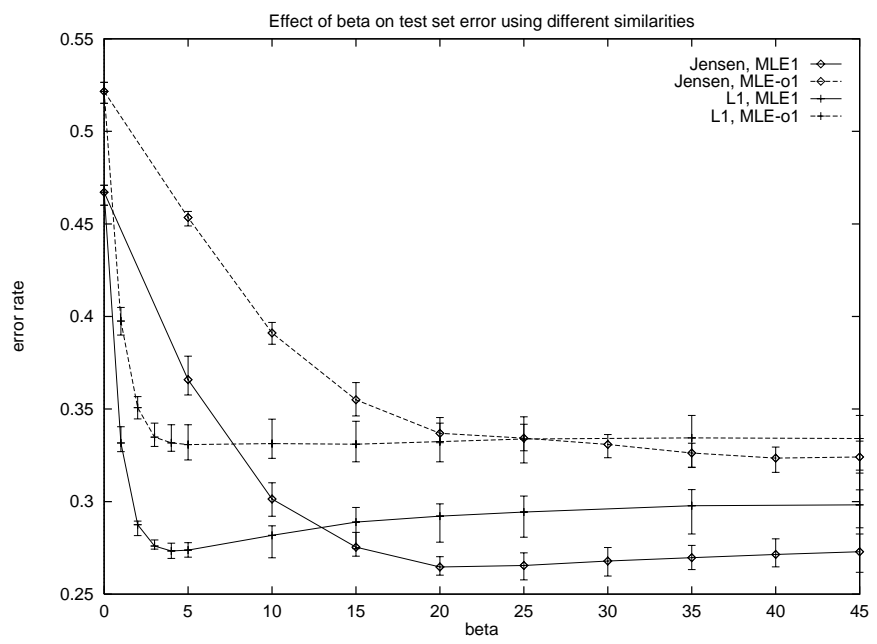

Figure 3. Average and range of test-set error rates as $\beta$ is varied. The similarity function is indicated by the point style; the base language model is indicated by the line style.

value is chosen, setting $\beta$ suboptimally does not greatly impact performance. Furthermore, the shape of the curves is the same for both base language models, suggesting that the relation between $\beta$ and test-set performance is relatively insensitive to variations in training data.

The fact that higher values of $\beta$ seem to lead to better error rates suggests that $\beta$ 's role is to filter out distant neighbors. To test this hypothesis, we experimented with using only the $k$ most similar neighbors. Figure 4 shows how the error rate depends on $k$ for different fixed values of $\beta$. The two lowest curves depict the performance of the Jensen-Shannon divergence and the $L_{1}$ norm when $\beta$ is set to the optimal value with respect to average test set performance; it appears that the more distant neighbors have essentially no effect on error rate because their contribution to the sum (9) is negligible. In contrast, when too low a value of $\beta$ is chosen (the upper two curves), distant neighbors are weighted too heavily. In this case, including more distant neighbors causes serious degradation of performance.

Interestingly, the behavior of the confusion probability is different from these two cases: adding more neighbors actually improves the error rate. This seems to indicate that the confusion probability is not correctly ranking similar words in order of informativeness. However, an alternative explanation is that $P_{\mathrm{C}}$ is at a disadvantage only because it is not being employed in the context of a tunable weighting scheme.

To distinguish between these two possibilities, we ran an experiment that dispensed with weights altogether. Instead, we took a vote of the $k$ most similar neighbors: the alternative chosen as more likely was the one preferred by a majority of the most similar neighbors (note that we ignored the degree to which alternatives were preferred). The results are shown in Figure 5.

We see that the $k$ most similar neighbors according to $J$ and $L$ were always more informative than those chosen according to the confusion probability, with the largest performance 


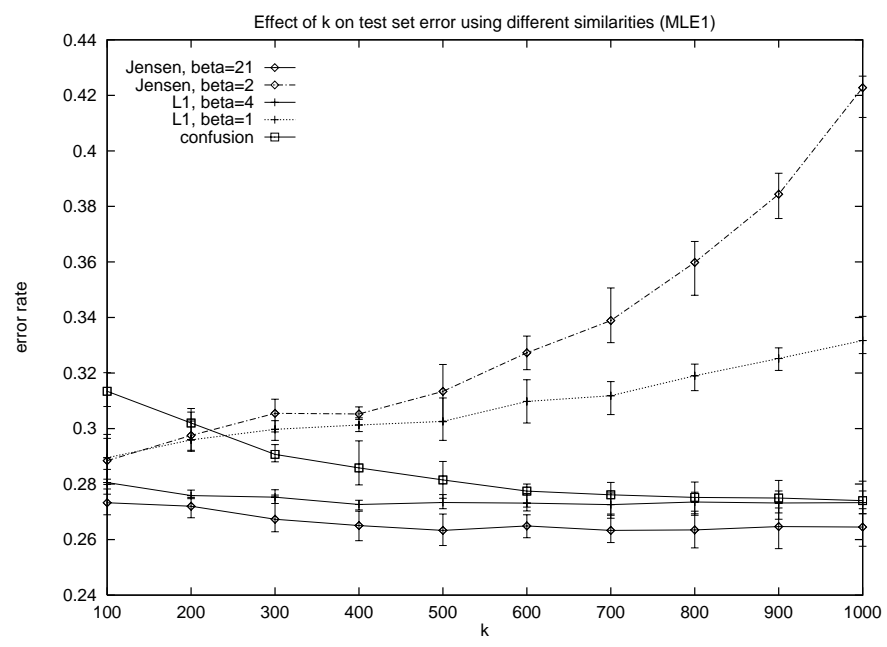

Figure 4. Average and range of test-set error rates as $k$ is varied. The base language model was MLE-1. The similarity function is indicated by the point style; the dashed and dotted lines indicate a suboptimal choice of $\beta$.

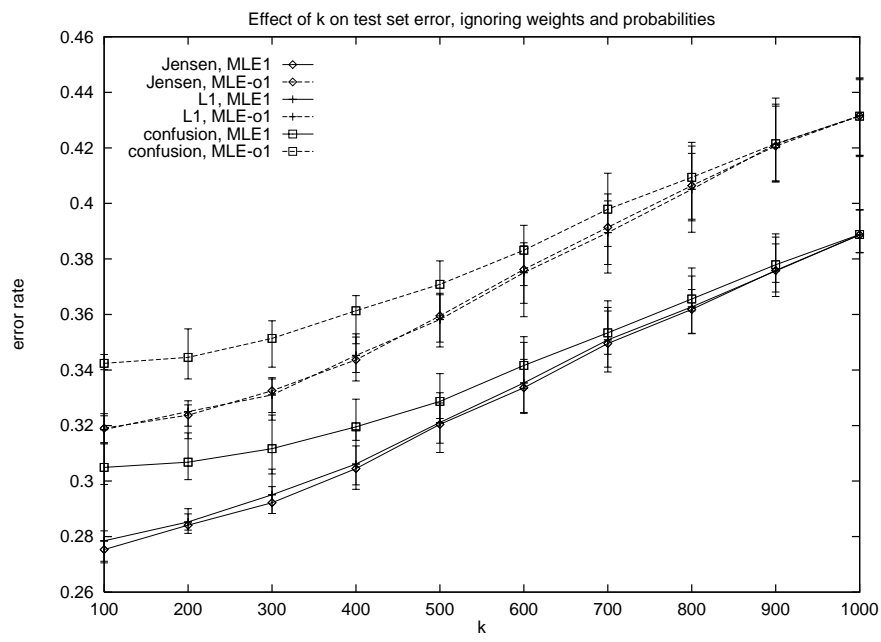

Figure 5. Average and range of voting-scheme test-set error rates as $k$ is varied. The similarity function is indicated by the point style; the base language model is indicated by the line style.

gaps occurring for low $k$ (of course, all methods performed the same for $k=1000$, since in that case they were using the same set of neighbors). This graph provides clear evidence that the confusion probability is not as good a measure of the informativeness of other words. 


\section{Related Work}

There is a large body of work on notions of work similarity, word clustering, and their applications. It is impossible to compare all those methods directly, since the assumptions, experimental settings and applications of methods vary widely. Therefore, the discussion below is mainly descriptive, highlighting some of the main similarities and differences between the methods.

\subsection{Statistical similarity and clustering for disambiguation and language modeling}

Our work is an instance of a growing body of research on using word similarity to improve performance in language-processing problems. Similarity-based algorithms either use the similarity scores between a word and other words directly in making their predictions, or rely on similarity scores between a word and representatives of precomputed similarity classes.

An early attempt to automatically classify words into semantic classes was carried out in the Linguistic String Project (Grishman, Hirschman, \& Nhan, 1986). Semantic classes were derived from similar cooccurrence patterns of words within syntactic relations. Cooccurrence statistics were then considered at the class level and used to alleviate data sparseness in syntactic disambiguation.

Schütze (1992b, 1993) captures contextual word similarity by first reducing the dimensionality of a context representation using singular value decomposition and then using the reduced-dimensionality representation to characterize the possible contexts of a word. This information is used for word sense disambiguation. All occurrences of an ambiguous word are clustered and each cluster is mapped manually to one of the senses of the word. The context vector of a new occurrence of the ambiguous word is mapped to the nearest cluster which determines the sense for that occurrence. Schütze emphasizes that his method avoids clustering words into a pre-defined set of classes, claiming that such clustering is likely to introduce artificial boundaries that cut off words from part of their semantic neighborhood.

Karov \& Edelman (1996) have also addressed the data sparseness problem in word sense disambiguation by using word similarity. They use a circular definition for both a word similarity measure and a context similarity measure. The circularity is resolved by an iterative process in which the system learns a set of typical usages for each of the senses of an ambiguous word. Given a new occurrence of the ambiguous word the system selects the sense whose typical context is most similar to the current context, applying a procedure which resembles the sense selection process of Shütze.

Our scheme for employing word similarity in disambiguation was influenced by the work of Dagan et al. (1993, 1995). Their method computes a word similarity measure directly from word cooccurrence data. A word is then modeled by a set of most similar words, and the plausibility of an unseen cooccurrence is judged by the cooccurrence statistics of the words in this set. The similarity measure is a weighted Tanimoto measure, a version of which was also used by Grefenstette $(1992,1994)$. Word association is measured by mutual information, following earlier work on word similarity by Hindle (1990).

The method of Dagan et al. does not provide probabilistic models. Disambiguation decisions are based on comparing scores for different alternatives, but they do not produce 
explicit probability estimates and therefore cannot be integrated directly within a larger probabilistic framework. The cooccurrence smoothing model of Essen \& Steinbiss (1992), like our model, produces explicit estimates of word cooccurrence probabilities based on the cooccurrence statistics of similar words. The similarity-based estimates are interpolated with direct estimates of $n$-gram probabilities to form a smoothed $n$-gram language model. Word similarity in this model is computed by the confusion probability measure, which we described and evaluated earlier.

Several language modeling methods produce similarity-based probability estimates through class-based models. These methods do not use a direct measure of the similarity between a word and other words, but instead cluster the words into classes using a global optimization criterion. Brown et al. (1992) present a class-based $n$-gram model which records probabilities of sequences of word classes instead of sequences of individual words. The probability estimate for a bigram which contains a particular word is affected by bigram statistics for other words in the same class, where all words in the same class are considered similar in their cooccurrence behavior. Word classes are formed by a bottom-up hard-clustering algorithm whose objective function is the average mutual information of class cooccurrence. Ushioda (1996) introduces several improvements to mutual-information clustering. His method, which was applied to part-of-speech tagging, records all classes which contained a particular word during the bottom-up merging process. The word is then represented by a mixture of these classes rather than by a single class.

The algorithms of Kneser \& Ney (1993) and Ueberla (1994) are similar to that of Brown et al. (1992), although a different optimization criterion is used, and the number of clusters remains constant throughout the membership assignment process. Pereira et al. (1993) use a formalism from statistical mechanics to derive a top-down soft-clustering algorithm with probabilistic class membership. Word cooccurrence probability is then modeled by a weighted average of class cooccurrence probabilities, where the weights correspond to membership probabilities of words within classes.

\subsection{Thesaurus-based similarity}

The approaches described in the previous section induce word similarity relationships or word clusters from cooccurrence statistics in a corpus. Other researchers developed methods which quantify similarity relationships based on information in the manually crafted WordNet thesaurus (Miller, Beckwith, Fellbaum, Gross, \& Miller, 1990). Resnik (1992, 1995) proposes a node-based approach for measuring the similarity between a pair of words in the thesaurus and applies it to various disambiguation tasks. His similarity function is an information-theoretic measure of the informativeness of the least general common ancestor of the two words in the thesaurus classification. Jiang \& Conrath (1997) combine the node-based approach with an edge-based approach, where the similarity of nodes in the thesaurus is influenced by the path that connects them. Their similarity method was tested on a data set of word pair similarity ratings derived from human judgments.

Lin (1997, 1998) derives a general concept-similarity measure from assumptions on desired properties of similarity. His measure is a function of the number of bits required to describe each of the two concepts as well as their "commonality". He then describes an 
instantiation of the measure for a hierarchical thesaurus and applies it to WordNet as part of a word sense disambiguation algorithm.

\subsection{Contextual similarity for information retrieval}

Query expansion in information retrieval (IR) provides an additional motivation for automatic identification of word similarity. One line of work in the IR literature considers two words as similar if they occur often in the same documents. Another line of work considers the same type of word similarity we are concerned with, that is, similarity measured derived from word-cooccurrence statistics.

Grefenstette $(1992,1994)$ argues that cooccurrence within a document yields similarity judgements that are not sharp enough for query expansion. Instead, he extracts coarse syntactic relationships from texts and represents a word by the set of its word-cooccurrences within each relation. Word similarity is defined by a weighted version of the Tanimoto measure which compares the cooccurrence statistics of two words. The similarity method was evaluated by measuring its impact on retrieval performance.

Ruge (1992) also extracted word cooccurrences within syntactic relationships and evaluated several similarity measures on those data, focusing on versions of the cosine measure. The similarity rankings obtained by these measures were compared to those produced by human judges.

\section{Conclusions}

Similarity-based language models provide an appealing approach for dealing with data sparseness. In this work, we proposed a general method for using similarity-based models to improve the estimates of existing language models, and we evaluated a range of similaritybased models and parameter settings on important language-processing tasks. In the pilot study, we compared the language modeling performance of a similarity-based model with a standard back-off model. While the improvement we achieved over a bigram back-off model is statistically significant, it is relatively modest in its overall effect because of the small proportion of unseen events. In a second, more detailed study we compared several similarity-based models and parameter settings on a smaller, more manageable word-sense disambiguation task. We observed that the similarity-based methods perform much better on unseen word pairs, with the measure based on the Jensen-Shannon divergence being the best overall.

Our experiments were restricted to bigram probability estimation for reasons of simplicity and computational cost. However, the relatively small proportion of unseen bigrams in test data makes the effect of similarity-based methods necessarily modest in the overall tasks. We believe that the benefits of similarity-based methods would be more substantial in tasks with a larger proportion of unseen events, for instance language modeling with longer contexts. There is no obstacle in principle to doing this: in the trigram case, for example, we would still be determining the probability of pairs $V_{1} \times V_{2}$, but $V_{1}$ would consist of word pairs instead of single words. However, the number of possible similar events to a given element in $V_{1}$ is then much larger than in the bigram case. Direct tabulation of the events most similar to each event would thus not be practical, so more compact or approximate representations 
would have to be investigated. It would also be worth investigating the benefit of similaritybased methods to improve estimates for low-frequency seen events. However, we would need to replace the back-off model by another one that combines multiple estimates for the same event, for example an interpolated model with context-dependent interpolation parameters.

Another area for further investigation is the relationship between similarity-based and class-based approaches. As mentioned in the introduction, both rely on a common intuition, namely, that events can be modeled to some extent by similar events. Class-based methods are more computationally expensive at training time than nearest neighbor methods because they require searching for the best model structure (number of classes and, for hard clustering, class membership) and estimation of hidden parameters (class membership probabilities in soft clustering). On the other hand, class-based methods reduce dimensionality and are thus smaller and more efficient at test time. Dimensionality reduction has also been claimed to improve generalization to test data, but the evidence for this is mixed. Furthermore, some class-based models have theoretically satisfying probabilistic interpretations (Saul \& Pereira, 1997), whereas the justification for our similarity-based models is heuristic and empirical at present. Given the variety of class-based language modeling algorithms, as described in the section on related work above, it is beyond the scope of this paper to compare the performance of the two approaches. However, such a comparison, especially one that would bring both approaches under a common probabilistic interpretation, would be well worth pursuing.

\section{Acknowledgments}

We thank Hiyan Alshawi, Joshua Goodman, Rebecca Hwa, Slava Katz, Doug McIlroy, Stuart Shieber, and Yoram Singer for many helpful discussions, Doug Paul for help with his bigram back-off model, and Andrej Ljolje and Michael Riley for providing word lattices for our speech recognition evaluation. We also thank the reviewers of this paper for their constructive criticisms, and the editors of the present issue, Claire Cardie and Ray Mooney, for their help and suggestions. Portions of this work have appeared previously (Dagan, Pereira, \& Lee, 1994; Dagan, Lee, \& Pereira, 1997); we thank the reviewers of those papers for their comments. Part of this work was done while the first author was a member of technical staff and then a visitor at AT\&T Labs, and the second author was a graduate student at Harvard University and a summer visitor at AT\&T Labs. The second author received partial support from the National Science Foundation under Grant No. IRI-9350192, a National Science Foundation Graduate Fellowship, and an AT\&T GRPW/ALFP grant.

\section{Notes}

1. To the best of our knowledge, this is the first use of this particular distribution dissimilarity function in statistical language processing. The function itself is implicit in earlier work on distributional clustering (Pereira et al., 1993) and has been used by Tishby (p.c.) in other distributional similarity work. Finch (1993) discusses its use in word clustering, but does not provide an experimental evaluation on actual data.

2. We experimented with using $10^{-\beta L\left(w_{1}, w_{1}^{\prime}\right)}$ as well, but it yielded poorer performance results. 
3. Actually, they present two alternative definitions. We use their model 2-B, which they found yielded the best experimental results.

4. The ARPA WSJ development corpora come in two versions, one with verbalized punctuation and the other without. We used the latter in all our experiments.

5. Values of $\beta$ and $t$ refer to base 10 logarithms and exponentials in all calculations.

6. It should be noted, however, that on BO-1 data, the KL-divergence performed slightly better than the $L_{1}$ norm.

\section{References}

Aha, D. W., Kibler, D., \& Albert, M. K. (1991). Instance-based learning algorithms. Machine Learning, 6, 37-66.

Atkeson, C. G., Moore, A. W., \& Schaal, S. (1997). Locally weighted learning. Artificial Intelligence Review, 11(1), 11-73.

Brown, P. F., DellaPietra, V. J., deSouza, P. V., Lai, J. C., \& Mercer, R. L. (1992). Class-based $n$-gram models of natural language. Computational Linguistics, 18(4), 467-479.

Cardie, C. (1993). A case-based approach to knowledge acquisition for domain-specific sentence analysis. In 11th National Conference on Artifical Intelligence (pp. 798-803). Menlo Park, California: AAAI.

Chen, S. F., \& Goodman, J. T. (1996). An empirical study of smoothing techniques for language modeling. In $34 t h$ Annual Meeting of the ACL (p. 310-318). Somerset, New Jersey: Association for Computational Linguistics. (Distributed by Morgan Kaufmann, San Francisco)

Church, K. W. (1988). A stochastic parts program and noun phrase parser for unrestricted text. In Second Conference on Applied Natural Language Processing (p. 136-143). Somerset, New Jersey: Association for Computational Linguistics. (Distributed by Morgan Kaufmann, San Francisco)

Church, K. W., \& Gale, W. A. (1991). A comparison of the enhanced Good-Turing and deleted estimation methods for estimating probabilities of English bigrams. Computer Speech and Language, 5, 19-54.

Cover, T. M., \& Hart, P. (1967). Nearest neighbor pattern classification. IEEE Transactions on Information Theory, 13(1), 21-27.

Cover, T. M., \& Thomas, J. A. (1991). Elements of information theory. New York: John Wiley.

Dagan, I., Lee, L., \& Pereira, F. C. N. (1997). Similarity-based methods for word sense disambiguation. In 35th Annual Meeting of the ACL (pp. 56-63). Somerset, New Jersey: Association for Computational Linguistics. (Distributed by Morgan Kaufmann, San Francisco)

Dagan, I., Marcus, S., \& Markovitch, S. (1993). Contextual word similarity and estimation from sparse data. In 31st Annual Meeting of the ACL (pp. 164-171). Somerset, New Jersey: Association for Computational Linguistics. (Distributed by Morgan Kaufmann, San Francisco)

Dagan, I., Marcus, S., \& Markovitch, S. (1995). Contextual word similarity and estimation from sparse data. Computer Speech and Language, 9, 123-152.

Dagan, I., Pereira, F. C. N., \& Lee, L. (1994). Similarity-based estimation of word cooccurrence probabilities. In 32nd Annual Meeting of the ACL (p. 272-278). Somerset, New Jersey: Association for Computational Linguistics. (Distributed by Morgan Kaufmann, San Francisco)

Devroye, L., Györfi, L., \& Lugosi, G. (1996). A probabilistic theory of pattern recognition. New York: Springer-Verlag.

Duda, R. O., \& Hart, P. E. (1973). Pattern classification and scene analysis. New York: Wiley-Interscience.

Essen, U., \& Steinbiss, V. (1992). Co-occurrence smoothing for stochastic language modeling. In ICASSP 92 (Vol. 1, p. 161-164). Piscataway, New Jersey: IEEE.

Finch, S. (1993). Finding structure in language. Unpublished doctoral dissertation, University of Edinburgh.

Gale, W., Church, K. W., \& Yarowsky, D. (1992). Work on statistical methods for word sense disambiguation. In R. Goldman (Ed.), Fall Symposium on Probabilistic Approaches to Natural Language (pp. 54-60). Menlo Park, California: AAAI.

Good, I. J. (1953). The population frequencies of species and the estimation of population parameters. Biometrika, $40(3$ and 4$), 237-264$.

Grefenstette, G. (1992). Use of syntactic context to produce term association lists for text retrieval. In International conference on research and development in information retrieval, SIGIR (p. 89-97). New York: ACM.

Grefenstette, G. (1994). Explorations in automatic thesaurus discovery. Boston: Kluwer Academic Publishers.

Grishman, R., Hirschman, L., \& Nhan, N. T. (1986). Discovery procedures for sublanguage selectional patterns - initial experiments. Computational Linguistics, 12, 205-214. 
Grishman, R., \& Sterling, J. (1993). Smoothing of automatically generated selectional constraints. In Human Language Technology: Proceedings of the ARPA Workshop (pp. 254-259). San Francisco: Morgan Kaufmann.

Hindle, D. (1990). Noun classification from predicate-argument structures. In 28th Annual Meeting of the ACL (pp. 268-275). Somerset, New Jersey: Association for Computational Linguistics. (Distributed by Morgan Kaufmann, San Francisco)

Jelinek, F., \& Mercer, R. L. (1980). Interpolated estimation of Markov source parameters from sparse data. In Proceedings of the Workshop on Pattern Recognition in Practice. Amsterdam: North Holland.

Jelinek, F., Mercer, R. L., \& Roukos, S. (1992). Principles of lexical language modeling for speech recognition. In S. Furui \& M. M. Sondhi (Eds.), Advances in speech signal processing (pp. 651-699). New York: Marcel Dekker.

Jiang, J. J., \& Conrath, D. W. (1997). Semantic similarity based on corpus statistics and lexical taxonomy. In ROCLING X International Conference. Tapei, Taiwan: Academia Sinica.

Karov, Y., \& Edelman, S. (1996). Learning similarity-based word sense disambiguation from sparse data. In E. Ejerhed \& I. Dagan (Eds.), Fourth Workshop on Very Large Corpora (pp. 42-55). Somerset, New Jersey: Association for Computational Linguistics.

Katz, S. M. (1987). Estimation of probabilities from sparse data for the language model component of a speech recognizer. IEEE Transactions on Acoustics, Speech and Signal Processing, ASSP-35(3), 400-401.

Kneser, R., \& Ney, H. (1993). Improved clustering techniques for class-based statistical language modelling. In EUROSPEECH'93 (pp. 973-976). Grenoble, France: European Speech Communication Association.

Kullback, S. (1959). Information theory and statistics. New York: John Wiley and Sons.

Lee, L. (1997). Similarity-based approaches to natural language processing. Unpublished doctoral dissertation, Harvard University, Cambridge, Massachusetts.

Lin, D. (1997). Using syntactic dependency as local context to resolve word sense ambiguity. In 35th Annual Meeting of the ACL (pp. 64-71). Somerset, New Jersey: Association for Computational Linguistics. (Distributed by Morgan Kaufmann, San Francisco)

Lin, D. (1998). An information theoretic definition of similarity. In Machine Learning: Proceedings of the Fiftheenth International Conference (ICML '98). San Francisco: Morgan Kaufmann.

Lin, J. (1991). Divergence measures based on the Shannon entropy. IEEE Transactions on Information Theory, 37(1), 145-151.

Luk, A. K. (1995). Statistical sense disambiguation with relatively small corpora using dictionary definitions. In 33rd Annual Meeting of the ACL (pp. 181-188). Somerset, New Jersey: Association for Computational Linguistics. (Distributed by Morgan Kaufmann, San Francisco)

Miller, G. A., Beckwith, R., Fellbaum, C., Gross, D., \& Miller, K. J. (1990). Introduction to WordNet: an online lexical database. International Journal of Lexicography, 3(4), 235-244.

$\mathrm{Ng}, \mathrm{H}$. T. (1997). Exemplar-based word sense disambiguation: Some recent improvements. In C. Cardie \& R. Weischedel (Eds.), Second Conference on Empirical Methods in Natural Language Processing (EMNLP-2) (pp. 208-213). Somerset, New Jersey: Association for Computational Linguistics.

$\mathrm{Ng}, \mathrm{H}$. T., \& Lee, H. B. (1996). Integrating multiple knowledge sources to disambiguate word sense: An exemplar-based approach. In 34th Annual Meeting of the ACL (pp. 40-47). Somerset, New Jersey: Association for Computational Linguistics. (Distributed by Morgan Kaufmann, San Francisco)

Paul, D. B. (1991). Experience with a stack decoder-based HMM CSR and back-off n-gram language models. In ARPA Speech and Natural Language Workshop (pp. 284-288). San Francisco: Morgan Kaufmann.

Pereira, F. C. N., Tishby, N., \& Lee, L. (1993). Distributional clustering of English words. In 31 st Annual Meeting of the ACL (p. 183-190). Somerset, New Jersey: Association for Computational Linguistics. (Distributed by Morgan Kaufmann, San Francisco)

Rao, C. R. (1982). Diversity: Its measurement, decomposition, apportionment and analysis. Sankyhā: The Indian Journal of Statistics, 44(A), 1-22.

Resnik, P. (1992). Wordnet and distributional analysis: A class-based approach to lexical discovery. In Workshop on Statistically-based Natural Language Processing Techniques (p. 56-64). Menlo Park, California: AAAI.

Resnik, P. (1995). Disambiguating noun groupings with respect to WordNet senses. In D. Yarowsky \& K. W. Church (Eds.), Third Workshop on Very Large Corpora (pp. 54-68). Somerset, New Jersey: Association for Computational Linguistics.

Ruge, G. (1992). Experiments on linguistically-based term associations. Information Processing \& Management, 28(3), 317-332.

Saul, L., \& Pereira, F. C. N. (1997). Aggregate and mixed-order Markov models for statistical language processing. In C. Cardie \& R. Weischedel (Eds.), Second Conference on Empirical Methods in Natural Language Processing (EMNLP-2) (pp. 81-89). Somerset, New Jersey: Association for Computational Linguistics. 
Schütze, H. (1992a). Context space. In R. Goldman (Ed.), Fall Symposium on Probabilistic Approaches to Natural Language (pp. 113-120). Menlo Park, California: AAAI.

Schütze, H. (1992b). Dimensions of meaning. In Supercomputing '92: Proceedings of the ACM/IEEE Conference (p. 787-796). New York: ACM.

Schütze, H. (1993). Word space. In S. J. Hanson, J. D. Cowan, \& C. L. Giles (Eds.), Advances in Neural Information Processing Systems 5 (pp. 895-902). San Francisco: Morgan Kaufmann.

Stanfill, C., \& Waltz, D. (1986). Toward memory-based reasoning. Communications of the ACM, 29(12), 1213-1228.

Sugawara, K., Nishimura, M., Toshioka, K., Okochi, M., \& Kaneko, T. (1985). Isolated word recognition using hidden Markov models. In ICASSP 85 (pp. 1-4). Piscataway, New Jersey: IEEE.

Ueberla, J. P. (1994). An extended clustering algorithm for statistical language models (Tech. Rep. No. DRA/CIS(CSE1)/RN94/13). Forum Technology - DRA Malvern.

Ushioda, A. (1996). Hierarchical clustering of words and applications to NLP tasks. In E. Ejerhed \& I. Dagan (Eds.), Fourth Workshop on Very Large Corpora (pp. 28-41). Somerset, New Jersey: Association for Computational Linguistics.

Witten, I. H., \& Bell, T. C. (1991). The zero-frequency problem: Estimating the probabilities of novel events in adaptive text compression. IEEE Transactions on Information Theory, 37(4), 1085-1094.

Yarowsky, D. (1992). Word-sense disambiguation using statistical models of Roget's categories trained on large corpora. In COLING-92 (pp. 454-460). Nantes, France.

Zavrel, J., \& Daelemans, W. (1997). Memory-based learning: Using similarity for smoothing. In 35th Annual Meeting of the ACL (pp. 436-443). Somerset, New Jersey: Association for Computational Linguistics. (Distributed by Morgan Kaufmann, San Francisco) 